\title{
Instruments et territoires de la gouvernance des déchets au Liban
}

Instruments and spaces of waste governance in Lebanon

Jihad Farah et Éric Verdeil

\section{(2) OpenEdition}

Journals

Édition électronique

URL : http://journals.openedition.org/geocarrefour/16766

DOI : $10.4000 /$ geocarrefour. 16766

ISSN : 1960-601X

Éditeur

Association des amis de la Revue de géographie de Lyon

Référence électronique

Jihad Farah et Éric Verdeil, «Instruments et territoires de la gouvernance des déchets au Liban », Géocarrefour [En ligne], 95/1 | 2021, mis en ligne le 23 février 2021, consulté le 23 février 2021. URL http://journals.openedition.org/geocarrefour/16766; DOI : https://doi.org/10.4000/geocarrefour. 16766

Ce document a été généré automatiquement le 23 février 2021.

(c) Géocarrefour 


\title{
Instruments et territoires de la gouvernance des déchets au Liban
}

\author{
Instruments and spaces of waste governance in Lebanon
}

\author{
Jihad Farah et Éric Verdeil
}

Les auteurs remercient Emilio Gemayel, Rasha Ghaddar, Elie Nasr, Rita Nasr, Hanan Wehbe pour leur contribution à l'enquête, et Rita Nasr plus particulièrement pour sa cartographie. Ils remercient également Patrick Le Galès pour ses commentaires sur une version antérieure du manuscrit et les deux réviseurs anonymes de la revue.

\section{Introduction}

1 Durant l'été 2015, des habitants mobilisés vivant près de la décharge de Naamé, au sud de Beyrouth, en ont bloqué l'accès afin de mettre un terme aux nuisances dues au manque de contrôle du stockage et à sa saturation. L'accumulation des déchets dans les rues a alors déclenché une crise politique majeure, qui a mis en évidence la mauvaise gestion du secteur par le gouvernement depuis la fin de la guerre civile en 1991 et la faible performance de Sukleen et Averda, les entreprises chargées de la collecte depuis 1994 et du stockage depuis 1997 à Beyrouth et au Mont Liban. Les manifestions, au-delà des craintes pour la santé des citoyens, ont dénoncé la corruption de la classe politique. La crise a également ouvert un débat sur l'autorité légitime pour la gestion des déchets. Depuis une loi datant de 1977, les municipalités sont légalement en charge de cette tâche. Mais depuis les années 1990, l'Etat l'a assumée dans la région capitale. Néanmoins, hors de cette région des municipalités ont continué à gérer les déchets, et d'autres ont revendiqué leur droit de le faire depuis la crise.

2 La crise des déchets au Liban apparaît comme un cas propre à soulever plusieurs questions identifiées par les coordinateurs de ce numéro dans leur appel à penser le politique par les déchets (Maccaglia et Cirelli 2019). D'abord, en offrant la possibilité de transformer le mode de gouvernement de la filière de gestion des déchets, la crise semble une occasion de renforcer le pouvoir des municipalités. La politisation de la 
gestion permettrait de renforcer la légitimité des pouvoirs locaux grâce à de nouvelles politiques présentées comme efficaces et/ou offrant des bénéfices locaux (amélioration de l'environnement, emplois). On peut s'interroger sur les formes de régulation politique qui apparaissent dans cette situation. Cette question se pose d'une part sur le plan de la transformation de l'action publique, et fait écho aux discussions sur la décentralisation et le rôle des municipalités par rapport aux fonctions assurées par le gouvernement. Elle renvoie d'autre part à des enjeux politiques dans lesquels les expériences municipales contribuent à la construction de rapports de force à l'échelle nationale. Enfin, un troisième enjeu concerne les relations d'affaires entre pouvoirs publics et opérateurs du secteur des déchets. Ce marché, régulé à travers des formes de contractualisation nouvelles que les pouvoirs locaux maitrisent mal, suscite une âpre compétition pour les profits qu'ils génèrent mais aussi des arrangements illicites, bénéficiant à des intérêts privés ou à des partis politiques.

Dans cet article, nous soutenons qu'à partir des choix effectués pour résoudre cette question de politique urbaine, les autorités locales peuvent s'autonomiser et renforcer leur légitimité à gouverner en favorisant l'émergence de communautés fonctionnelles d'intérêt local. Cependant, une telle évolution dépend de l'articulation des instruments d'action publique mis en place et des réseaux politiques mobilisés, dans des configurations d'échelle variable. Nous adaptons le cadre de la sociologie politique des instruments et de l'instrumentation de l'action publique à l'analyse du gouvernement de la filière des déchets solides municipaux. L'objectif est de voir dans quelle mesure et comment les politiques de gestion des déchets mises en œuvre dans trois municipalités de ce pays, Zahlé, Saïda et Bikfaya, ont été construites autour d'instruments convergents et stabilisés, organisant tant les relations entre autorités locales et citoyens que celles entre les autorités nationales et locales. L'article revient d'abord sur le cadre d'analyse utilisé, puis sur le contexte libanais, les cas d'étude mobilisés et les méthodes utilisés. Nous exposons ensuite les résultats de notre grille d'analyse

\section{Cadre d'analyse de la gouvernance locale des déchets}

Dans les recherches sur le politique à l'échelle urbaine, les modalités de gestion des services urbains sont le plus souvent analysées comme une variable dépendante de variables explicatives qui sont les luttes de pouvoir entre représentants de différents groupes sociaux, en particulier à l'occasion des compétitions électorales, et l'intervention de pouvoirs de niveaux supérieurs. Les services urbains sont souvent pensés comme un véhicule de relations clientélaires visant à la consolidation du pouvoir (Post 2018). Dans ces travaux, la question des déchets est rarement étudiée par rapport à d'autres services locaux (eau, transport, santé, éducation...). Surtout, faire des modes de gestion d'un service local comme les déchets une variable induisant des formes de régulations politiques du jeu politique locale est peu fréquent. Certains enjeux liés à la régulation des déchets donnent certes lieu à des formes de politisation : c'est le cas, notamment, de la question de l'emploi informel (Cavé 2015 ; Cirelli, Florin, Troin (éd.), 2015 ; De Bercegol, Cavé, et Durand 2018) ou de conflits liés aux nuisances occasionnées par de nouvelles installations (Demaria et Schindler 2016; Cirelli, Maccaglia, et Melé 2017; de Bercegol et Gowda 2018). Dans la plupart des cas, la diversité des acteurs publics est peu problématisée et les travaux se focalisent le plus souvent sur le gouvernement central, acteur majeur des réformes successives de la 
filière déchets, à travers la promotion des partenariats public-privé ou plus récemment de l'introduction de nouvelles logiques de valorisation (Jaglin, Debout, Salenson (éd.), 2018). Le gouvernement local est généralement perçu négativement, comme faiblement doté en compétences et ressources (Katusiimeh, Mol, et Burger 2012; Okot-Okumu et Nyenje 2011; Fakoya 2014). Cette situation d'insuffisance technique et institutionnelle est fréquemment renvoyée à une forme d'arriération culturelle qui fait des municipalités des lieux de relations politiques primordiales fermées à la modernité (pour une critique de ce type dans le cadre régional, mais sans référence explicite au secteur des déchets, voir Salman and Baird-Zars, 2019). A l'inverse, nous proposons une perspective dynamique insistant sur la construction du pouvoir local à partir du déploiement et la gestion du service des déchets.

Nous utilisons pour ce faire la sociologie de l'instrumentation pour évaluer la construction sur le long terme d'instruments de politiques publiques visant à réguler la gestion des déchets à l'échelle locale (Knoepfel, Larrue, et Varone 2006 ; Lascoumes, Le Galès (éd.), 2004 ; Lascoumes et Simard 2011 ; Halpern, Lascoumes, Le Galès (éd.), 2014). Dans cette perspective, l'instrument est « un type particulier d'institution, un dispositif technique ayant pour objet générique de porter une conception concrète de la relation politico-sociétale et soutenu par une notion de régulation " (Lascoumes et Le Galès, 2004). Nous nous attachons à comprendre le processus politique qui sous-tend la manière dont les processus techniques et opérationnels sont décidés et intégrés dans les structures institutionnelles existantes. Il s'agit d'étudier une gouvernance des déchets, c'est à dire "un processus d'agrégation, de coordination et de direction d'acteurs, de groupes sociaux et d'organisations, en vue d'atteindre des objectifs définis et discutés collectivement. » (Le Galès, 2019, p. 298). Dans ce cadre, nous posons deux questions : comment la gouvernance des déchets à l'échelle locale, entendue comme l'articulation de plusieurs régulations incarnées par des instruments (Le Galès, 2019, p. 303), est-elle produite et stabilisée dans le temps ? Comment cette gouvernance des déchets permet-elle de conforter des modes de relations de pouvoir existantes ou permet-elle d'en construire d'autres?

6 Pour les besoins de cet article, nous nous concentrons sur les types d'instruments que les autorités locales initient ou négocient avec les instances de gouvernement de niveau comparable ou supérieur, ainsi qu'avec les acteurs privés et avec la population, afin de gérer les déchets municipaux. Classiquement on distingue les instruments suivants : les contrats entre le secteur public et le secteur privé (choix de la technologie, durée, partage des risques et des investissements, suivi des performances); les instruments financiers et fiscaux (taxes, transferts de l'État) et les instruments utilisés pour communiquer avec la population et la mobiliser (notamment à l'échelle des quartiers). Les acteurs municipaux mobilisent également des instruments qui ont pour particularité leur rapport au territoire: d'abord l'aménagement urbain, qui inclut le zonage et l'acquisition de terrains pour la mise en décharge et le traitement, et ensuite la définition du périmètre desservi par l'unité de traitement.

7 Nous examinons ces modes de régulation et de leur stabilisation en distinguant le rôle des acteurs et réseaux politiques, des contextes temporels et des spatialités. Pour une plus grande clarté analytique, nous distinguons ce qui relève spécifiquement de la régulation des déchets et ce qui structure plus largement le fonctionnement du politique à l'échelle locale, dans ses articulations avec les autres échelles. 
Tableau 1 : Facteurs contribuant à la stabilisation d'instruments de régulation de la gestion locale des déchets

\begin{tabular}{|c|c|c|c|}
\hline & Acteurs et réseaux... & Spatialités ... & Temporalités... \\
\hline $\begin{array}{l}\ldots \quad \mathrm{du} \\
\text { politique }\end{array}$ & $\begin{array}{l}\text { - Partis et grands } \\
\text { leaders politiques } \\
\text { nationaux } \\
\text { - Puissances } \\
\text { régionales et } \\
\text { internationales } \\
\text { - Monde économique } \\
\text { - Institutions } \\
\text { publiques } \\
\text { - Notables locaux } \\
\text { - Réseaux d'activistes } \\
\text { de la société civile } \\
\text { - Médias }\end{array}$ & $\begin{array}{l}\text { - Spatialités } \\
\text { environnementales } \\
\text { (écosystèmes, bassins } \\
\text { versants, littoral, } \\
\text { réserves, aquifères, etc.) } \\
\text { - Territoires administratifs } \\
\text { - Territoires identitaires } \\
\text { (communautaires, } \\
\text { politiques) } \\
\text { - Intercommunalités } \\
\text { fonctionnelles } \\
\text { (notamment pour services } \\
\text { urbains) }\end{array}$ & $\begin{array}{l}\text { - Les élections } \\
\text { - Les crises politiques } \\
\text { nationales } \\
\text { régionales }\end{array}$ \\
\hline $\begin{array}{l}\ldots \text { des } \\
\text { déchets }\end{array}$ & $\begin{array}{l}\text { - Autorités locales } \\
\text { - Ministères et } \\
\text { institutions } \\
\text { publiques } \\
\text { concernées } \\
\text { - Bailleurs de fonds } \\
\text { - Entreprises } \\
\text { d'implantation des } \\
\text { équipements de } \\
\text { déchets et de leur } \\
\text { opération } \\
\text { - Activistes et ONGs } \\
\text { locales }\end{array}$ & $\begin{array}{l}\text { - Spatialité des nuisances } \\
\text { - Spatialité des flux de } \\
\text { collecte et de traitement } \\
\text { des déchets }\end{array}$ & $\begin{array}{l}\text { - Rythmes et capacité de } \\
\text { traitement des déchets } \\
\text { - Temporalités des } \\
\text { contrats (durée, litiges, } \\
\text { etc.) } \\
\text { - Temporalités des } \\
\text { financement (appels à } \\
\text { projets, transferts du } \\
\text { Fonds autonome des } \\
\text { municipalités, etc.) } \\
\text { - Durée de vie des } \\
\text { infrastructures } \\
\text { (saturation, limite de } \\
\text { vie, etc.) }\end{array}$ \\
\hline
\end{tabular}

Nous relions ensuite ce travail de spécification de la gouvernance locale des déchets à trois types d'enjeux politiques qui, particulièrement saillants au Liban, ont une portée plus générale. Premièrement, l'autonomisation du local par rapport au gouvernement renvoie au débat sur la décentralisation. Malgré les dispositions de la loi libanaise octroyant de nombreuses prérogatives aux municipalités, en pratique leur autonomisation gestionnaire est entravée à la fois par la permanence d'un contrôle à priori des actes et une forte limitation des moyens disponibles. Ce contrôle administratif renvoie au maintien de mécanismes de domination des pouvoirs locaux par les acteurs politiques de premier plan se partageant l'accès aux ressources étatiques et leurs redistributions (Favier (éd.), 2001; Harb et Atallah, 2015). Un deuxième enjeu concerne la construction de la légitimité politique politique. Elle peut dériver de pratiques clientélistes (distribution d'emplois par exemple) (Post, 2018), ou s'appuyer sur un sentiment d'appartenance collectif structuré par une identité religieuse ou locale (Catusse, Karam, et Lamlūm, 2011; Mermier et Mervin, 2012). La légitimation de l'action publique peut passer aussi par l'affichage d'une efficacité technocratique dans la résolution des problèmes (Kadirbeyoğlu et Sümer 2012 ; Carles, 2018). Un troisième enjeu a trait à la construction du territoire comme espace local ou 
régional de solidarité et de coopération (Dubresson et Jaglin, 2005). Des coopérations fonctionnelles sur base territoriale permettent-elles une structuration politique durable? Dans la suite de ce travail, et à travers trois cas, nous explorons ces assemblages d'instruments, montrant leurs effets politiques pour des autorités locales s'attaquant à la question des déchets.

\section{Collecte des données}

9 Le choix des études de cas a été effectué à travers une revue de presse couvrant la période 1998-2017 (1700 articles) et une compilation de sources publiques sur la gestion des déchets à l'échelle municipale. L'analyse des terrains s'est fait en deux phases : d'abord, une enquête confiée à des étudiants stagiaires a documenté les principaux aspects institutionnels et techniques des gestions municipales, et un premier aperçu des contestations locales (14 entretiens et observations). Puis les auteurs ont complété l'analyse des modes de régulation de la gestion des déchets en menant une dizaine d'entretiens complémentaires élargissant le spectre des acteurs rencontrés et en croisant ces informations avec la presse et les analyses académiques disponibles (notamment dans le cas de Saïda). Une présentation plus détaillée du recueil des données est présentée en annexe.

Cette recherche ne repose pas sur une ethnographie des jeux politiques en actes autour de la gestion des déchets mais sur une photographie sur une période courte eut égard à l'histoire relativement longue de ces projets. La documentation secondaire, notamment la presse, représente une source majeure d'informations. L'étude des cas est menée avec une perspective diachronique et en insistant sur les moments favorisant la cristallisation ou du moins la stabilisation relative des instruments. Nous analysons cette institutionnalisation des instruments en lien avec des négociations, des rapports de force et des conflits reconstitués par le croisement des diverses sources d'information et lues en lien avec la bibliographie existante sur les rapports de pouvoir au Liban.

\section{La crise des déchets au Liban : étapes, cadre d'interprétation et sélection des études de cas}

11 La crise des déchets qui se déroule au Liban depuis 2015 ne peut être comprise qu'à la lumière des politiques publiques mises en œuvre au sortir de la guerre civile, en réponse à une première série de tensions culminant en 1994 et 1997. Déjà, les ordures non ramassées dans les rues de Beyrouth et de sa banlieue suscitent des manifestations, parfois violentes, des populations riveraines des décharges improvisées et des infrastructures de traitement, saturées et polluantes (Huybrechts et Verdeil, 2000). Les solutions adoptées alors déterminent l'architecture du système qui prévaut pendant vingt ans. Le Conseil du développement et de la reconstruction octroie en 1994 une délégation de service public à la société Sukleen pour la collecte des déchets à Beyrouth, puis confie à une filiale du même groupe (Sukomi) l'aménagement et la gestion d'une grande décharge située à Naameh, au sud de la capitale, et d'installations de tri et de production de compost. La licence de Sukleen est graduellement étendue et couvre bientôt la totalité du Mont Liban à l'exception du caza de Jbeil (Boutros, 2015). Dans les autres régions, où les décharges sauvages ont proliféré pendant la guerre et les 
premières années qui la suivent, la gestion des déchets reste une responsabilité municipale mais le gouvernement intervient, en liaison avec des aides internationales centralisées par le CDR, pour aménager des décharges sanitaires, en général confiées par les municipalités à des sociétés sous-traitantes. Cette situation perdure globalement jusqu'en 2015. Néanmoins, la presse évoque des controverses politiques concernant le cout des contrats de Sukleen, beaucoup plus élevé que ceux des autres régions, et le faible niveau des prestations de recyclage et de compostage. Elle souligne aussi les connexions étroites qui lient le groupe Averda (propriétaire de Sukleen) et la famille Hariri, qui occupe un rôle politique majeur sur toute la période et contrôle en particulier le CDR (Rozelier, 2010 ; Rozelier, 2018). Dans le contexte de la crise de 2015, d'autres sources indiquent que d'autres politiciens de premier plan sont également actionnaires de cette société (Middle East Strategic Perspectives, 2015). Malgré ces soupçons étalés sur la place publique, les contrats dont bénéficie le groupe AVERDA sont renouvelés à plusieurs reprises, alimentant les rumeurs d'un cartel de corruption (Zbeed, 2012).

12 Le déclenchement de la crise en 2015 a plusieurs conséquences politiques : d'une part, la contestation du gouvernement prend une dimension inédite et élargie à d'autres revendications (services publics, corruption, déconfessionalisation). Les manifestations, violemment réprimées, fragilisent le gouvernement mais les groupes d'activistes se divisent (Kraidy, 2016; AbiYaghi, Catusse et Younes, 2017; El Richani, 2017 ; Harb, 2018). Après moult péripéties, le contrat de Sukleen et d'Averda est interrompu. Le gouvernement attribue le marché de la collecte à une nouvelle société, à un tarif peu différent tandis qu'il ouvre des décharges en mer, sur le site d'anciens dépôts d'ordures. Présentées comme des solutions temporaires, leur exploitation a été prolongée et leurs surfaces étendues à plusieurs reprises (Baaklini, 2020). Elles ont des conséquences environnementales dramatiques tout en mettant en évidence l'incapacité de trouver des solutions durables tant au niveau de la réduction du volume de déchets (par le tri à la source) que pour le traitement et le recyclage, avec de grosses controverses sur la construction d'incinérateurs et le stockage final dans des lieux restants à identifier (Baaklini, 2019).

Durant cette période, 18 municipalités de la région du Mont Liban ont récupéré la prérogative de gestion des déchets, et lancent des initiatives variées: politiques d'encouragement du tri, recyclage, compostage, partenariats avec des ONG ou des industriels pour développer des solutions d'incinération, parfois en associant plusieurs municipalités voisines (figure $\mathrm{n}^{\circ} 1$ ). 
Figure 1 : Gestion des déchets municipaux au Liban, zones desservies et projets en 2018

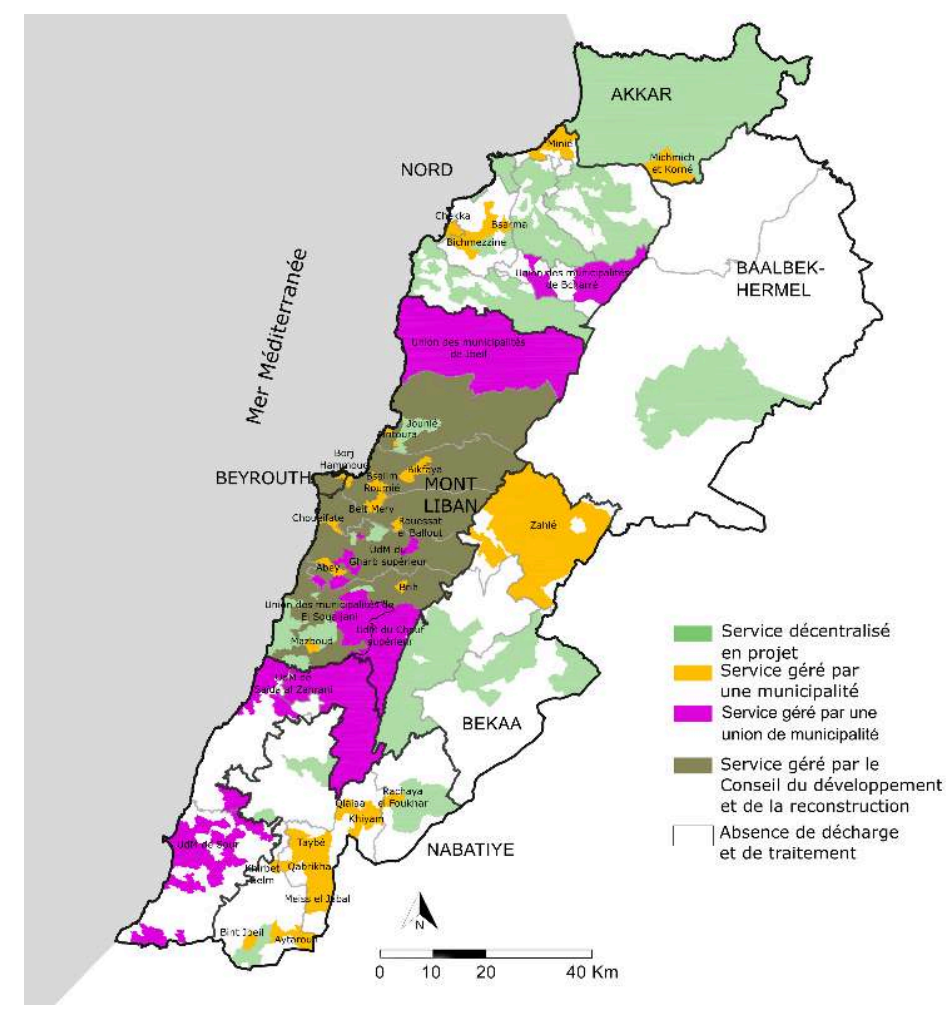

Cartographie : Rita Nasr ; Source : Enquête CERDA auprès des municipalités et des gestionnaires des entreprises, 2018

Ces dernières années, le Parlement a adopté deux lois qui visent à introduire de nouvelles pratiques dans la gestion des déchets et la prestation de services publics en général : la loi 48/2017 sur les partenariats public-privé (PPP) et la loi 80/2018 sur la gestion intégrée des déchets. Cette dernière est le résultat d'un long processus piloté par le ministère de l'Environnement avec l'aide du PNUD. Elle vise à établir un cadre réglementaire qui pousserait vers des solutions de gestion des déchets plus intégrées articulant la réduction, la réutilisation, le recyclage, le traitement et la mise en décharge des déchets. Néanmoins, ces lois ont été critiquées par les partisans d'une décentralisation plus forte ainsi que par les associations environnementalistes (Abi Akl 2018). En effet, elles renforcent le rôle des autorités centrales au détriment des autorités locales. En outre, elles ne s'intéressent pas suffisamment aux systèmes de recouvrement des coûts, ne favorisent pas l'introduction d'une fiscalité locale des déchets et placent beaucoup d'espoir dans les systèmes de partenariat public-privé. Enfin, elles demandent de nombreux décrets exécutifs, ce qui n'est pas en phase avec l'urgence des problèmes de gestion des déchets au Liban.

La crise de la gestion des déchets à Beyrouth a été très largement commentée, avec deux orientations principales. La première, technique et normative, est centrée sur les critiques des plans gouvernementaux et la proposition de systèmes plus efficaces, notamment sur le plan environnemental (Ghadban, Shames, et Abou Mayaleh, 2017; Human Rights Watch, 2017 ; Morsi, Safa, Baroud, Fawaz, Farha, El-Jardali et Chaaya, 2017 ; Hammoud, Kassem, et Mourtada, 2014 ; Karaki, 2016 ; Abbas, Chaaban, Al-Rabaa, et Shaar, 2017 ; Azzi, 2017 ; Massoud, Mokbel, Alawieh, et Yassin, 2019). Globalement, ces travaux négligent la diversité des expérimentations techniques au niveau local. La 
seconde approche, relevant davantage de l'économie politique critique, souligne les liens entre la corruption dans le secteur des déchets et la domination de la classe politique divisée en partis confessionnels. Le rôle central du Fonds autonome des municipalités est notamment analysé : les taxes dues aux municipalités, collectées par des organismes nationaux et redistribuées à ces dernières sur des bases très opaques, sont utilisées pour rémunérer les opérateurs choisis par le CDR pour collecter les déchets et gérer les décharges après des appels d'offre peu transparents (Atallah, 2015a ; Atallah, 2015b ; Atallah, 2015c ; Abu Rish, 2015 ; Chaaban, 2017). Cette littérature se concentre sur Beyrouth et sa banlieue immédiate, ou adopte un point de vue national et ne met donc pas en évidence la diversité des modes de gestion des déchets à travers le pays. C'est pourquoi notre article se concentre sur l'échelon local et a sélectionné trois cas représentatifs de la diversité des choix politiques effectués.

\section{Trois situations, trois assemblages différents d'instruments}

Dans ce qui suit nous discutons les cas de Zahlé, Saïda et Bikfaya, en présentant leur contexte territorial, sociopolitique, l'évolution de la gouvernance locale des déchets, notamment la mobilisation des différents instruments de politique publique et ses répercussions sur les principaux enjeux politiques des autorités locales.

\section{Zahlé}

17 Zahlé est la principale ville de la région de la Beqaa, au centre d'une grande plaine agricole. Elle compte 72000 habitants au sein d'une agglomération de 150000 habitants. L'économie de la ville repose historiquement sur un équilibre entre centre de services, commerce et agriculture. Dans les dernières décennies l'activité industrielle se renforce $d u$ fait du faible coût du foncier. La population de la ville même est composée très majoritairement de chrétiens de divers rites, dans une agglomération mixte avec une forte présence de populations chiites et sunnites respectivement au nord et au sud.

Depuis les années soixante, Zahlé a connu trois sites successifs de décharges pour déchets solides. La deuxième décharge était arrivée à saturation à la fin des années 1990. La municipalité construisit à partir de 2000 dans la plaine agricole, loin des zones bâties une décharge sanitaire, une plate-forme pour le tri des déchets et des installations de compostage. Ces installations furent inaugurées en 2007 (Bluhm, 2007). Cet ensemble dessert 24 autres municipalités qui y transportent leurs ordures. Le développement de la décharge représente un jalon essentiel de la consolidation de la capacité de la municipalité et surtout son président à s'imposer sur le plan politique.

\section{Un réseau d'acteurs « a-politiques »}

19 Zahlé était historiquement dominée par des notables claniques, à l'écart des partis politiques communautaires. Avant la guerre civile (1975-1990), ces notables de Zahlé contrôlaient au Parlement un bloc de députés issus de l'ensemble de la Bekaa, qui leur conféraient un poids décisif dans la politique nationale. Dans l'après-guerre, les notables et clans familiaux de la ville ont progressivement perdu leur influence dans la 
Bekaa et au sein des institutions étatiques. Leurs divisions croissantes les ont mis dans la dépendance des principales coalitions politiques du Liban après 2005, dites du 8 et du 14 Mars, menées dans les communautés chrétiennes par les partis du Courant Patriotique Libre et des Forces Libanaises (Chaoul, 2012). Les clans familiaux ont subi une défaite totale face à ces partis lors des élections municipales de 2016 et législatives de 2018.

Ce contexte éclaire la carrière du maire actuel de Zahlé, Assaad Zoughaib. Arrivé à la mairie en 1998 avec le soutien du clan zahliote des Skaf, il y resté jusqu'en 2010 lorsque ce clan lui a préféré un autre candidat. En 2016, il redevient maire, cette fois soutenu par une coalition de partis. Cependant, ingénieur de formation, il se dit technocrate non-politisé. Dans le contexte libanais, cela signifie qu'il n'est pas affilié aux grands partis communautaires nationaux. Il préconise à l'échelle locale une politique qui met en avant les questions de "développement»: efficacité des services publics et des infrastructures, soutien aux activités économiques, amélioration de la qualité de l'espace urbain et protection de l'environnement.

Cet effort de développement nécessite des ressources considérables. Or, si les municipalités des grandes villes comme Zahlé peuvent compter sur une diversité de taxes locales (sur les nouvelles constructions, les logements et les entreprises, etc.) pour financer leur budget, elles ont aussi besoin des transferts du Fonds autonome des municipalités (FAM) pour équilibrer leurs revenus et leurs dépenses ${ }^{1}$. Mais l'irrégularité des versements du FAM et des règles de répartition des fonds mouvantes rendent difficiles la planification des investissements à partir de ces ressources seules. Depuis les années 1990, les organisations internationales et bilatérales de développement (dont la Banque mondiale, l'Union européenne et l'US Aid) y ont pourvu en fournissant 111 millions de dollars de subventions et de prêts à faible taux d'intérêt au Liban pour faire face à la situation problématique des déchets, notamment pour des décharges sanitaires et, plus tard, des installations de tri et de traitement ${ }^{2}$. Malgré de récurrentes controverses sur l'efficacité de ces projets financés par les institutions internationales (voir par exemple Jay, 2019), ces fonds ont été cruciaux dans le développement de nombreuses installations, y compris à Zahlé. Leur obtention nécessite un savoir-faire pour la rédaction de projets ainsi qu'un capital social de relations avec les responsables de ces organisations internationales ou des institutions $\mathrm{du}$ gouvernement central, en particulier le Conseil du développement et de la reconstruction (CDR) et le Bureau du ministre de la réforme administrative (OMSAR) qui assurent la liaison avec elles. La maitrise par le maire et son équipe de cet espace relationnel et technique et la reconnaissance de leur capacité dans la mise en œuvre et la gestion des projets financés leur assurent un accès privilégié à ces fonds ${ }^{3}$. Ainsi, le site a été progressivement équipé de nouveaux équipements pour le recyclage et le compostage avec des financements de US Aid. Un suivi strict des entreprises en charge du service, y compris via l'application de sanctions en cas de contre-performance, a assuré un fonctionnement efficace contribuant à cette reconnaissance par les financeurs, mais aussi la population.

Le mode de gouvernance des déchets mis en place à Zahlé repose sur un réseau d'acteurs marqué par leur profil technique et un refus de s'appuyer sur les citoyens. Dans nos entretiens, le maire a exprimé son scepticisme quant à l'implication citoyenne dans une affaire qui pour lui est avant tout technique et gestionnaire. Citant l'échec d'une expérience de tri à la source lancée par la municipalité dans un des quartiers il 
affirme que l'implication citoyenne pourrait marcher à l'échelle d'un village mais pas dans les grandes villes. D'autre part, le maire n'a pas non plus fait appel aux réseaux d'acteurs politiques pour soutenir les projets concernant les déchets, afin d'éviter toute récupération dans les controverses politiques locales. Ceci se reflète dans la forme et le contenu de la communication relative à ces réalisations. Le maire est souvent invité à des conférences et séminaires dans les réseaux de coopération au développement local au Liban pour partager l'expérience de Zahlé, de même qu'il organise des visites pour des délégations de municipalités venant de tout le pays (Al Binaa, 2018). Il y souligne toujours la capacité des autorités locales à agir indépendamment de l'État central et des tensions politiques en adoptant une approche qualifiée de "professionnelle " et en s'impliquant pour maîtriser les aspects techniques du projet.

\section{Collaboration territoriale régionale et maîtrise foncière}

La mise à distance des conflits et clivages politiques se manifeste également dans les rapports entre Zahlé et son entourage dans la gestion des déchets. Dans une Bekaa centrale où les identités confessionnelles se sont consolidées dans les dernières décennies et où les partis communautaires sont à la conquête des postes de députés et des conseils municipaux, la concorde qui règne en matière de gestion des déchets ne va pas de soi. Les localités comprises dans le périmètre de collecte de déchets de la station de traitement de Zahlé n'appartiennent pas à une même union municipale 4 et ont des populations d'appartenance confessionnelle et des tendances politiques diverses. De plus, la relation de la municipalité de Zahlé avec l'Union des Municipalités du Caza de Zahlé à laquelle elle appartient est difficile car elle refuse de se retrouver sur un pied d'égalité avec des municipalités villageoises lors du vote des projets. De ce fait, elle a alterné dans les deux dernières décennies retraits et retours comme membre de l'Union. Le choix de la municipalité d'élargir le périmètre de collecte à 24 localités illustre le poids de la question environnementale pour le maire. Sans être écologiste, il a toujours soutenu l'importance de la protection de l'environnement pour la qualité de vie à Zahlé mais aussi pour la protection de l'agriculture qui représente toujours un poids économique conséquent dans l'économie de la ville. Les pratiques de décharges sauvages et de brûlage des déchets des municipalités voisines polluent lourdement un environnement partagé (eaux souterraines, rivières et atmosphère) avec le chef-lieu de la Bekaa ${ }^{5}$. Pour encourager ces localités à utiliser la décharge municipale, la municipalité leur a proposé des coûts de traitement très bas (autour de $10 \$ /$ tonne entrante en 2007) ne couvrant de fait que les coûts opérationnels. Toutefois, il serait erroné de lire dans cette extension du périmètre des déchets une volonté politique de construction de territoire inclusif à l'échelle de la Bekaa centrale centré sur la municipalité et son maire. À l'inverse, les positions des acteurs politiques à Zahlé, y compris au sein du conseil municipal, reflètent une tendance générale au «repli» communautaire.

Par ailleurs, le cas de Zahlé illustre la crise assez commune des sites de décharge sanitaire. Toutes les deux décennies environ, les sites successifs font face à deux défis : ils sont rattrapés par l'urbanisation et deviennent saturés par les déchets. À la fin des années 1990, l'achat par la municipalité du terrain actuel a été considéré comme une décision stratégique. Le bas prix du foncier dans la plaine agricole isolée à la frontière municipale a permis d'acheter un très grand terrain. Les deux anciennes décharges ont été réaménagées et l'une d'entre elles est devenu un parc urbain. La taille du terrain a 
également permis à la municipalité de Zahlé d'accepter les déchets de municipalités voisines. Cependant, au rythme actuel de remplissage, la municipalité estime que la décharge sera saturée en 2023. L'urbanisation rapide de ces dernières décennies a presque atteint la zone du site et le prix des terrains augmente, ce qui rend financièrement et politiquement difficile pour la municipalité une extension sur place sans parler de la remplacer par une nouvelle installation sur le territoire municipal. La municipalité a récemment refusé de recevoir les déchets de nouvelles municipalités de la région pour freiner le rythme de saturation du site. Elle envisage actuellement l'adoption d'une technologie qui pourrait l'émanciper du besoin de nouveaux terrains pour la mise en décharge. Dans nos entretiens en 2017 et 2018, le maire mettait en avant une solution basée sur l'incinération et un partenariat public-privé. Or, ce choix technologique pourrait générer des mobilisations de contestation citoyennes, comme ça été le cas pour les projets d'incinérateurs à Beyrouth et ailleurs au Liban, et par suite mettre fin à « l'a-politisme » de la gouvernance des déchets portée par la municipalité à Zahlé depuis deux décennies. Plus récemment, la municipalité semble plus réticente d'aller dans cette voie et cherche encore d'autres solutions alternatives ${ }^{6}$.

\section{La mise en boîte noire et l'anticipation pour maîtriser les temporalités}

La gestion des déchets à Zahlé n'a pas été affectée par les périodes électorales locales ni par les crises au niveau national. Même la crise des déchets de 2015 qui a obligé beaucoup d'autorités locales au Liban à s'impliquer dans la gestion des déchets, n'a eu aucune conséquence directe à Zahlé. De même, si les élections municipales de 2010 ont mené à la mise à l'écart de A. Zoughaib pendant 6 ans, la gestion des déchets n'a pas été modifiée. La stabilisation de cette organisation est le produit d'un assemblage d'instruments qui a permis d'assurer une régulation pérenne des relations contractuelles, des financements, du fonctionnement du site et de la collecte des déchets - tout ceci accompagné par une communication bien huilée qui vante la gestion des déchets à Zahlé comme une réussite ${ }^{7}$. Mais la saturation de la décharge attendue en 2023 pourrait remettre en cause cette situation. Le choix d'une nouvelle technologie présente le risque de rouvrir la «boîte noire " de traitement de déchets à Zahlé, alors que l'équilibre du nouvel assemblage d'instruments reste à identifier.

\section{Saïda}

Saïda est une ville côtière de 65000 habitants dans un district qui en compte 250 000. La ville est le centre de la troisième agglomération du pays, le chef-lieu du gouvernorat du Sud-Liban, et un centre de services et de commerces. Elle comprend aussi une grande zone industrielle. La population de la ville appartient majoritairement à la communauté sunnite, alors que les banlieues de l'agglomération qui s'étalent sur les villages des piémonts sont plutôt chrétiennes et chiites.

La question des déchets a longtemps été une véritable plaie de la politique municipale à Saïda. La décharge de la ville s'est étendue sur des déblais de construction déposés sur la côte suite à l'invasion israélienne de 1982. Devenue une "montagne » de plusieurs dizaines de mètres de hauteur, elle polluait la mer et l'air, dégradant la qualité de vie dans les quartiers au sud de la ville et entravant le tourisme. À partir de 2010, la décharge a été traitée et nivelée en parc et, sur une partie de ce site, une entreprise privée a commencé à traiter les déchets de tout le district (Abou Afla, 2016). Elle trie les 
déchets, transforme la partie organique selon un procédé de biodigestion produisant de l'énergie, et vend les autres composants à des entreprises de recyclage. L'autorité officiellement responsable est l'Union des municipalités de Saïda-AlZahrani, dont le président est le maire de Saïda. Quoique présentée comme une solution efficace, la nouvelle usine de traitement des déchets est devenue elle-même une source de nuisances et est au centre d'une controverse environnementale mettant en cause la politique municipale sur ce plan et plus largement.

\section{Expérimentation controversée d'un partenariat public-privé très politique}

Saïda est le fief de la famille Hariri, dont les chefs Rafik puis Saad et leur allié Fouad Siniora ont été présidents du Conseil des ministres pendant la plus grande partie des trois dernières décennies. Bahia Hariri, sœur de Rafik, est députée de la ville depuis 2000. Toutefois, d'autres forces politiques sont historiquement implantées à Saida, notamment l'Organisation Populaire Nassérienne, résistent à l'emprise sur la ville du clan Hariri et de son parti le Mouvement du Futur. De fait, ce n'est qu'en 2010 que la mairie est passée aux mains du Mouvement du Futur et ses alliés. Ainsi l'arrivée de Mohammad Saoudi, un homme d'affaire proche du clan Hariri, à la tête de la municipalité vient consolider les transformations engagées à Saïda par Rafik Hariri depuis sa présidence du Conseil des ministres en 1992 (Bonne, 2014). La politique municipale historiquement conservatrice, dominée par le mouvement nassérien, privilégiant la satisfaction des besoins des populations défavorisées, connait un tournant. Pour rendre la ville plus attractive pour les classes moyennes et les investisseurs, la municipalité entreprend de grands projets d'aménagement et d'infrastructures : remembrement de la plaine agricole, aménagement et piétonisation du centre-ville pour attirer le tourisme, projets de nouveau front de mer, etc. (Moussi, 2014; Makhzoumi et Al-Sabbagh, 2018). C'est dans cet ensemble que s'inscrit la nouvelle unité de traitement des déchets solides à Saida.

La résolution de la question des déchets et surtout le traitement de la «montagne des déchets » constituent les priorités de la nouvelle équipe, afin de légitimer son action en démontrant leur efficacité (Al Moustaqbal, 2016), à l'opposé de ses opposants politiques n'ont pas réussi à résoudre la situation pendant plus d'une décennie. Pour s'attaquer à ce grand projet, le nouveau maire peut compter sur les ressources de plusieurs institutions et acteurs. Le traitement de la décharge a été rendu possible par un don saoudien de $20 \mathrm{M}$ \$. En 2012, sa transformation en parc en remblai sur la mer est achevée (Zaatari, 2012; Baaklini, 2016; Wehbe, 2012). Le CDR a assuré la maîtrise d'ouvrage des travaux. Quant à l'usine de traitement, elle est construite sur un terrain public gagné par sur la mer par IBC, une entreprise privée appartenant majoritairement à un entrepreneur de Saïda affilié au clan Hariri, et associé à des partenaires saoudiens. Le contrat d'IBC est signé avec l'union de municipalités présidée par le maire de la ville.

Tous ces acteurs et institutions impliqués sont affiliés ou contrôlés par le réseau Hariri. Pour autant, les relations entre eux n'ont pas toujours été des plus simples. La question $\mathrm{du}$ financement du contrat de l'entrepreneur est emblématique et révèle la situation de faiblesse et de dépendance de la municipalité par rapport à son patron politique. Le montage de ce projet est inédit au Liban et il n'existe pas de contrat type qui aurait pu être utilisé comme référence. Le contrat initial, signé en 1999 entre IBC, la municipalité et l'union, amendé en 2009, prévoyait la mise à disposition du terrain par la 
municipalité 8 , un investissement par l'entrepreneur privé de 25 millions de dollars US et le traitement gratuit de 200 tonnes/jour de déchets pour une durée de vingt ans. Les revenus attendus devaient provenir de la revente des produits recyclables et de l'énergie produite, ainsi que du tarif pour les déchets dépassant le seuil de $200 \mathrm{t} / \mathrm{j}$. Cependant, après avoir effectué les premiers investissements mais avant le début des opérations, IBC a affirmé que les projections des études de faisabilité économique ne concordaient plus avec la réalité, en raison d'une humidité des déchets plus forte que prévue et de l'activité des ramasseurs de déchets qui détournaient les déchets les plus précieux pour les recycler, entrainant une perte de revenu pour l'opérateur. Conformément aux stipulations du contrat initial, il s'est alors retiré et a demandé à l'union des municipalités d'exploiter directement le service. Cela était impossible en raison de son manque d'expertise et de financement (Chamseddine, 2018). Après près de deux ans de blocage, les parties sont parvenues en 2013 à un nouvel accord parrainé par le clan Hariri qui impliquant une fourniture minimale de 350 tonnes par jour et le paiement par l'union d'une redevance de 80 puis 95 US\$ par tonne (The Monthly, 2015 ; Baaklini, 2012). Cette situation illustre une nette dissymétrie dans le rapport de force entre les autorités publiques locales et l'acteur privé. Ce dernier a pu amener l'Union des municipalités à signer un contrat qui était beaucoup moins favorable à celle-ci que prévu initialement. Au-delà du défaut d'expertise technique, cette situation illustre également le manque de moyens juridiques et administratifs de la municipalité et de l'union et leur forte dépendance au clan politique, limitant leur capacité à concevoir un contrat protégeant l'intérêt public et contrôler le choix de la technologie. De fait, la municipalité et l'union s'appuyaient pour le conseil juridique sur un juriste de la Fondation Hariri (Chamseddine, 2018).

31 Ce défaut de ressources humaines concerne aussi l'aspect technique : la municipalité et l'union n'ont pas pu assurer un contrôle de la performance environnementale de l'usine qui s'est avéré très mauvaise, produisant des nuisances et suscitant des protestations. Cela malgré la désignation d'une entreprise de consultance par la municipalité pour assurer le respect du contrat en 2015 après une première mobilisation des associations et riverains (Khalil, 2015). Ces dernières culminèrent avec le blocage de l'installation par des manifestants durant l'été 2018 (OLJ, 2018 ; Antonios, 2018). Il a fait les gros titres pendant plus de dix ans (Baaklini, 2011 ; OLJ/agences, 2012). La municipalité et l'union laissent le propriétaire de l'installation occuper le premier plan du point de vue de la communication. Le discours structuré de l'entreprise, repris par la municipalité, se concentre sur les procédés innovants utilisés et la quantité considérable de déchets solides traités (Zantout, 2018; Zeater, 2015 ; Dahché, 2015; Al Charq, 2015) ${ }^{9}$. Malgré des similitudes avec la communication municipale à Zahlé dans le refus d'un engagement direct des citoyens et la confiance en la technologie, le discours tenu par les acteurs de Saida s'en distingue par l'importance accordée à l'initiative privée comme garantie d'innovation, de bonne gestion et d'efficacité. Cependant, le manque d'engagement de la population et de transparence a contribué à une situation de tension et de controverses, au sujet de la pollution marine et des nuisances et odeurs lors du remblaiement ou lors du stockage de déchets non traités pour cause de dépassement des capacités (Saleh, 2015; Abou Afla, 2016; Al Diyar, 2016; Khalil, 2018). Des riverains, des associations environnementales (notamment Min Ajl Baladi AlAkhdar (au nom d'un pays vert), Bahr Lubnan (La mer du Liban)) et les opposants politiques des Hariri attaquent les nuisances générées par l'usine, l'absence d'une politique en faveur du tri à la source, les pollutions, la 
privatisation des services ou encore le remblayage du front de mer. Tout ceci affaiblit la crédibilité de l'entreprise et de la municipalité pointée pour collusion.

\section{Territorialité métropolitaine et spatialité réticulaire des déchets}

32 Les ratés de la politique de traitement des déchets révèlent également des divergences entre les membres de l'Union des Municipalités de Saida-AlZahrani sur le futur du territoire et la répartition du fardeau des déchets. D'un côté, l'union est reconnue par les maires comme une institution légitime pour penser le futur du territoire, notamment à travers l'engagement d'une démarche d'un plan stratégique ${ }^{10}$. La tutelle de l'union sur l'usine de traitement participe également de la construction d'un territoire fonctionnel de référence. Néanmoins, la fermeture temporaire en 2018 d'une usine située dans la Bekaa jouant le rôle d'exutoire des résidus ultimes de l'installation d'IBC et l'impossibilité de vendre ces derniers comme combustibles aux cimenteries, comme prévu initialement, en raison de limitations réglementaires, a mis en évidence la nécessité de disposer d'un terrain d'enfouissement de ces matières. Des discussions houleuses au sein de l'union pour en identifier un dans une des localités membres n'ont pas abouti, affectant une solidarité territoriale fragile, obligeant à un stockage illégal dans un bassin non encore remblayé, entre le parc maritime et l'usine, ce qui engendré à nouveau des nuisances pestilentielles. Une décision de justice a interrompu ces dépôts, laissant pendante la question du stockage de ces matières (actuellement partiellement éliminées dans une usine de la Bekaa).

Cette question non résolue illustre plus largement les tensions et contradictions politiques qui résultent de la nature réticulaire de ce qu'on peut appeler le métabolisme des déchets, c'est-à-dire les dimensions spatiales de sa circulation entre lieu de production, de traitement et de stockage. D'une part, l'utilisation des déchets ultimes dans les opérations de réaménagement du littoral est lue par ses détracteurs, habitants, militants écologistes ou d'association civique comme LiSaida, ou opposants politiques ${ }^{11}$ comme un nouvel avatar des remblais aménagés sur la décharge du Normandy au centre-ville de Beyrouth, ou du projet Linord dans le Metn (Verdeil, 2017). Ils craignent un processus de transformation des déchets en foncier au profit des plus riches sans égard pour l'environnement et leur santé (Mansour, 2018). De fait, le slogan municipal "Changer la décharge en parc " illustre la volonté de transformer cette zone en un front de mer attractif. Mais au vu des incertitudes techniques, il est permis de penser que cette option ne pourra se matérialiser dans un avenir proche, illustrant un manque de maîtrise de la ville sur le devenir de son territoire.

La question de la circulation des déchets souligne la complexité des recompositions de la spatialité politique des déchets à Saïda. Pour assurer l'équilibre financier du projet, IBC doit en effet traiter une plus grande quantité de déchets que celle produite par les municipalités de l'union. Elle en importe d'autres régions du Sud Liban, notamment Jezzine. En 2015, en pleine crise dans la capitale, sous la pression du Mouvement du Futur, elle a aussi accepté de reprendre près de 250 tonnes/jour des déchets de Beyrouth, ce qui était une manière d'atténuer la tension dans cette ville qui constitue une autre base politique essentielle de ce parti ${ }^{12}$. Au contraire de Zahlé qui jusqu'à aujourd'hui a construit sa politique de gestion des déchets sur la base d'une coopération territoriale avec ses voisins, la politique menée à Saïda illustre l'émergence d'une spatialité réticulaire entre plusieurs régions du pays, non immunisée contre la toxicité perturbatrice des matières en circulation, ou en stagnation forcée. 


\section{Une temporalité dépendante du tempo d'un groupe politique} parti Kataeb (Phalanges) est actuellement dans l'opposition au gouvernement. Toutefois, si le Kataeb est dominant à Bikfaya, il est en forte compétition dans les autres localités du Metn avec d'autres partis communautaires chrétiens (Courant Patriotique Libre et Forces Libanaises), des grands notables régionaux (notamment l'ex-ministre et député Michel Murr) ou encore le parti anti-confessionnel PSNS. La stratégie de «reconquête » des Kataeb dans le district après le retrait des troupes syriennes du Liban s'est focalisée sur la réorganisation du parti au niveau local et la mobilisation pour les élections législatives et ensuite municipales. Sous la présidence de Sami Gemayel, fils de l'ancien président Amine, élu député du district, le parti se présente comme le champion de la décentralisation. Prouver que les municipalités sont en capacité de prendre en charge les déchets est pour lui un moyen de démontrer l'intérêt de la décentralisation (RAH, 2016). Nicole Gemayel est la sœur du chef du parti et son conseil municipal est dominé par les Kataeb.

La gouvernance des déchets à Saïda est soumise au rythme des temporalités politiques locales et nationales. De fait, après des années de gestation d'une solution et les péripéties infructueuses de l'équipe municipale précédente pour résoudre le problème des déchets à Saida, le projet s'est concrétisé avec l'arrivée d'une nouvelle équipe municipale soutenue par le réseau du clan Hariri en 2010, traduisant une recherche de légitimité basée sur l'efficacité. Bien que non directement concernée par la crise de 2015 , la ville y joue un rôle en tant qu'exutoire providentiel pour la capitale submergée par ses déchets. La gestion des déchets à Saida traduit les connexions entre l'agenda du Mouvement du Futur pour la ville et son action au niveau national, des connexions qui restent néanmoins instables.

\section{Bikfaya} nord-est de l'agglomération, dans le gouvernorat du Mont-Liban. Elle compte 20000 habitants avec les villages voisins mais la population estivale est beaucoup plus importante. La population est majoritairement chrétienne maronite. son contrat pour Beyrouth et le Mont-Liban. Lorsque la crise éclate, des militants locaux lancent l'initiative Biclean basée sur le tri, le recyclage des déchets et bientôt le compostage de la partie organique. Commencée de façon improvisée sur un terrain en zone urbaine, BiClean s'installe ensuite sur un terrain cédé par la municipalité dans la zone industrielle de Bikfaya. L'arrivée de Nicole Gemayel, principal animatrice de Biclean, à la tête de la municipalité depuis les élections de 2016, formalise l'initiative qui est reprise en main en régie par la municipalité.

\section{Réseau à ancrage local communautaire et politique}

Toutefois, au-delà du parti lui-même et de la municipalité dominée par le parti, Biclean réussit à mobiliser une grande partie des associations locales. En fait, le système de traitement des déchets de Bikfaya repose sur la capacité d'assurer des taux de tri 
primaire élevés afin de faciliter les processus de tri secondaire et de compostage. Pour cela, le réseau local d'organisations communautaires (scouts, paroisses, écoles religieuses), dont beaucoup sont affiliées ou influencées par la présence du parti Kataeb dans la ville, a joué un rôle central pour atteindre la population et maintenir la dynamique du tri. Cette mobilisation, basée sur le sentiment d'appartenance collective, joue aussi sur des mesures punitives (refus de collecte des sacs non-triés, amendes). BiClean bénéficie également du support technique procuré par des ONGs impliquées de longue date dans le recyclage au Liban. Elle profite à son lancement de financements et d'assistance de la coopération internationale (UKAID, Mercy Corps), avant de s'autonomiser.

40 Par l'usage très actif des réseaux sociaux et des visites régulières organisées dans les installations, les animateurs du projet insistent sur leur sens du Do-it-yourself, sur les vertus de la basse technologie et de l'engagement citoyen ${ }^{13}$. La municipalité de Bikfaya met en avant le succès de Biclean avec fierté et la maire promeut le projet dans les médias et dans des événements impliquant les réseaux d'acteurs du développement. Cet effort de communication autour du projet au niveau national est relayé par le parti mais dans une perspective politique notamment dans sa campagne pour une loi de décentralisation élargie (RAH 2016).

\section{Du local vers un projet de territorialisation politique... et retour au local}

41 La municipalité de Bikfaya fait partie de l'Union des Municipalités du Metn littoral et moyen, qui comprend une trentaine de municipalités. Elle est contrôlée par le clan des Murr dont le patriarche Michel Murr a été député et ministre. Lors de la crise de 2015, l'union a porté un projet d'incinérateur sur le littoral qui aurait servi tout le Metn (Akiki et Farfour, 2017). Mais le projet a été abandonné lorsque l'entreprise privée Ramco a été mandatée par le CDR pour la collecte et traitement des déchets des districts de Matn et Kesrwan. Bikfaya et Beit Meri sont les seules deux municipalités du Matn qui ont choisi de développer leurs propres solutions. Une autre municipalité de son agglomération, Sakiet El Misk-Bhersaf, s'est néanmoins associée au projet BiClean.

Dès son origine, la municipalité et le parti Kataeb ont souhaité à élargir l'initiative à d'autres localités du Metn. En 2017, la municipalité est entrée en négociations avec une société privée pour mettre en œuvre une technologie de valorisation énergétique des déchets, ce qui aurait impliqué d'associer 14 municipalités pour assurer un volume de déchets permettant de rentabiliser l'exploitation d'un incinérateur. Mais l'irrégularité de versements $d u$ Fonds autonome des municipalités, indispensable source de financement des projets municipaux, a rendu impossible l'engagement de ces municipalités et a même précipité le retrait de Sakiet El-Misk de BiClean en $2019^{14}$. Récemment, avec la crise économique et les difficultés de financer l'importation, les matières recyclées connaissent une hausse de leur cours. De même, sept municipalités du Metn sont entrées dans un accord avec Bikfaya pour lui envoyer des déchets recyclables sans pour autant briser leur contrat avec Ramco (Raidy, 2020). Tout ceci permet un meilleur équilibre financier pour Biclean. 


\section{La crise des déchets comme fenêtre d'opportunité politique mais un projet à la portée limitée}

Des trois cas étudiés, Bikfaya est celui où la politique et la crise nationales ont eu le plus d'impact sur la politique municipale et sur la gestion des déchets. La crise des déchets de 2015 a été le fait déclencheur du projet Biclean. Les élections municipales de 2016 viennent consolider le passage d'une initiative locale en politique municipale avec l'arrivée de Nicole Gemayel à la municipalité. Monté dans l'urgence, dans un esprit d'expérimentation et par le volontarisme d'une équipe soudée parvenant à mobiliser la population locale en jouant sur un fort sentiment d'appartenance, BiClean réussit à se stabiliser. L'impossibilité de monter en échelle, tant technologiquement que politiquement, principalement en raison des contraintes de financement pesant sur les municipalités, entrave l'ambition d'en faire une vitrine de la décentralisation prônée par le parti Kataeb et le ramène à sa dimension locale.

\section{Conclusions}

Cette section conclusive revient aux deux questions structurant cette recherche.

En premier lieu, nous nous sommes demandés comment (et jusqu'à quel point) la gouvernance des déchets a été stabilisée autour des instruments élaborés par les pouvoirs locaux. Les trois cas étudiés illustrent des combinaisons très diverses en ce qui concerne le poids de chaque instrument et son articulation avec les autres. Le tableau ci-dessous illustre la variété des instruments utilisés, de leurs articulations et leurs pondérations. Dans un contexte marqué par un Etat central peu prescripteur de règles et de normes dans ce domaine, et par la faiblesse des ressources financières et humaines, les municipalités locales effectuent des choix et des assemblages dont l'explication renvoie à des situations et des trajectoires spécifiques. Au-delà de la taille de la ville, de sa richesse économique et de la situation du territoire, retenons deux réponses à cette première question.

D'abord, la composition de ces assemblages et leur stabilisation (tableau 2) portent la marque des réseaux politiques de types très différents qui les ont façonnés :

- un réseau techniciste voire technocratique à Zahlé, trouvant des ressources dans l'aide internationale, pragmatiquement construit autour d'un territoire fonctionnel à l'échelle d'un bassin de vie ;

- un réseau soutenu par un mouvement national puissant à Saïda, capable de mobiliser des financements privés mais peinant à stabiliser son assise territoriale ;

- un réseau communautaire fortement ancré dans le territoire local, mais peinant à le dépasser et incapable de mobiliser des financements permettant de changer l'échelle des opérations à Bikfaya.

On observe ensuite, de manière transversale, le poids des contextes temporels dans la capacité de stabilisation, notamment le degré d'urgence dans lesquels sont effectués ces choix et les alternances politiques: anticipation à Zahlé, blocage et crise durable à Saïda, débloquée par l'alternance politique, non sans rebondissements; crise soudaine et émergence d'une équipe municipale d'une nouvelle génération à Bikfaya. 
Tableau 2 : Déclinaison et combinaison des instruments de politique publique

\begin{tabular}{|c|c|c|c|}
\hline & ZAHLÉ & SAIDA & BIKFAYA \\
\hline \multicolumn{4}{|c|}{ Instruments par type de politique publique } \\
\hline Contractuels (C) & $\begin{array}{l}\text { - Contrat de } \\
\text { management }\end{array}$ & $\cdot$ PPP & • Régie \\
\hline Financement (F) & $\begin{array}{l}\text { - Investissement: } \\
\text { Bailleurs } \\
\text { internationaux } \\
\text { - Opérations: MdZ } \\
\text { et municipalités } \\
\text { voisines } \\
\text { - Poids faible des } \\
\text { transferts de la } \\
\text { FAM }\end{array}$ & $\begin{array}{l}\text { - Investissement : Privé } \\
\text { - Opérations : MdS et } \\
\text { municipalités voisines } \\
\text { - Poids faible des } \\
\text { transferts de la FAM }\end{array}$ & $\begin{array}{l}\text { - Investissement et } \\
\text { opérations : local et } \\
\text { ensuite MdB } \\
\text { - Poids faible des } \\
\text { transferts de la } \\
\text { FAM }\end{array}$ \\
\hline $\begin{array}{l}\text { Engagement de } \\
\text { la population et } \\
\text { acteurs locaux } \\
\text { (E) }\end{array}$ & $\begin{array}{l}\text { - Pas d'engagement } \\
\text { - Communication } \\
\text { centrée sur } \\
\text { l'efficacité } \\
\text { technique }\end{array}$ & $\begin{array}{l}\text { - Pas d'engagement } \\
\text { - Communication } \\
\text { centrée sur l'efficacité } \\
\text { technique + rôle } \\
\text { positif de } \\
\text { l'entrepreneuriat } \\
\text { privé }\end{array}$ & $\begin{array}{ll}\text { - Mobilisation de } & \text { de } \\
\text { volontaires } & \text { et } \\
\text { d'associations } & \\
\text { locales } & \\
\text { - Communication } & \\
\text { centrée sur la } \\
\text { capacitation du } \\
\text { local }\end{array}$ \\
\hline $\begin{array}{l}\text { Aménagement } \\
\text { du territoire et } \\
\text { urbanisme (A) }\end{array}$ & $\begin{array}{l}\text { - La décharge sur } \\
\text { foncier municipal } \\
\text { loin de } \\
\text { l'urbanisation }\end{array}$ & $\begin{array}{l}\text { - L'usine au sein d'un } \\
\text { projet de remblayage } \\
\text { pour gain de foncier } \\
\text { sur mer }\end{array}$ & $\begin{array}{l}\text { - Le centre de tri } \\
\text { dans une zone } \\
\text { industrielle }\end{array}$ \\
\hline $\begin{array}{lr}\text { Périmètres } & \text { de } \\
\text { collecte } & \text { des } \\
\text { déchets }(P) & \end{array}$ & • Régional & - Multi-échelle & • Local \\
\hline \multicolumn{4}{|c|}{$\begin{array}{l}\text { Rôle respectif des instruments dans la (dé)stabilisation de la gouvernance locale } \\
\text { Les lettres font référence aux instruments dans le tableau ci-dessus }\end{array}$} \\
\hline $\begin{array}{l}\text { Structuration } \\
\text { des réseaux } \\
\text { d'acteurs }\end{array}$ & $\begin{array}{l}\text { - Techniciste sans } \\
\text { support politique }\end{array}$ & $\begin{array}{l}\text { - Clan politique } \\
\text { Monde d'affaires }\end{array}$ & $\begin{array}{l}\text { - Clan politique } \\
\text { Associatif }\end{array}$ \\
\hline Poids déterm. & • $(F)+(C)$ & $\cdot(F)+(C)$ & •(E) \\
\hline Spatialités & • Régionale & $\begin{array}{l}\text { - Régionale } \\
\text { - Axée sur le bassin } \\
\text { littoral } \\
\text { - Épousant } \\
\text { fluctuation des flux } \\
\text { des déchets }\end{array}$ & - Locale \\
\hline Poids déterm. & $\cdot(P)$ & $\cdot(P)+(A)+(F)$ & $\cdot(P)+(F)$ \\
\hline
\end{tabular}




\begin{tabular}{|c|c|c|c|}
\hline Temporalités & $\begin{array}{l}\text { - L'anticipation } \\
\text { pour maîtriser les } \\
\text { temporalités }\end{array}$ & $\begin{array}{l}\text { - Projection et } \\
\text { préparation mais } \\
\text { difficile adaptation }\end{array}$ & $\begin{array}{l}\text { - Réactivité, } \\
\text { volontarisme et } \\
\text { adaptabilité mais } \\
\begin{array}{l}\text { question de la } \\
\text { durabilité }\end{array}\end{array}$ \\
\hline Poids déterm. & (A) & - $(A)+(C)$ & (E) \\
\hline
\end{tabular}

\section{Gouvernances municipales locales et transformation des enjeux politiques}

La deuxième question de notre recherche porte sur la manière dont la gouvernance locale des déchets transforme les rapports de force politique, sur trois points en particulier : légitimation sur la scène politique locale et nationale; construction du territoire politique local ou régional; autonomisation par rapport aux institutions de l'État central.

\section{Déchets et légitimation politique}

À Zahlé, la capacité d'anticipation, la stabilisation des relations dans un réseau d'acteurs techniciste et dans un espace régional multicommunautaire et aux multiples affiliations politiques ont permis à la municipalité de Zahlé de faire reconnaitre sa gestion des déchets comme une réussite. Le succès de l'expérience de la gestion des déchets vient s'ajouter à celle récente d'Électricité de Zahlé. Cette entreprise privée est parvenue, dans un pays marqué par la crise du secteur de l'électricité et les coupures fréquentes, à assurer une fourniture continue et un service de bonne qualité à une quinzaine de localités dans la région de Zahlé (Verdeil, 2019). Dans un contexte actuel de forte contestation de la classe politique, tout ceci alimente au Liban l'image de Zahlé comme modèle où une gouvernance techniciste locale peut produire des solutions plus efficaces que celles portées par les partis et institutions centrales. Toutefois, la gestion des déchets reste dans cette ville une boîte noire pour la très grande majorité de la population. La boîte noire pourrait devenir une boîte de Pandore si la municipalité n'arrive pas à trouver une solution alternative à la décharge avant 2023.

On retrouve le même sentiment de fierté à Bikfaya. Toutefois, ici la question des déchets est plus présente dans le débat public et se présente comme une question essentielle et un défi permanent auquel le réseau d'acteurs de Bikfaya n'a le choix de répondre qu'avec une mobilisation continue. Cette mobilisation permet de souder et galvaniser une population largement affiliée aux Kataeb. À une échelle plus large, l'expérience fonctionne comme une vitrine légitimant la proposition de réformer la loi municipale vers plus de décentralisation comme défend le parti.

51 À Saïda, le réseau soutenant la municipalité élue en 2010 a dans un premier temps réussi son pari. Le démantèlement de la décharge littorale et l'ouverture de l'usine ont permis à la municipalité de Saïda et les partisans du clan Hariri de montrer qu'une politique d'aménagement plus volontariste et appuyée par des investissements privés pourrait projeter une autre image plus moderne de la ville. Toutefois, les controverses environnementales et les nuisances qui ont suivi ont brouillé cette image. La question 
des déchets devient ainsi un espace de contestation de l'équipe municipale et par ricochet, du clan Hariri.

\section{Déchets et territorialisation politique}

52 À première vue, la spatialité de la gestion des déchets semble favoriser une collaboration entre les espaces voisins, voire l'émergence d'un territoire local ou régional. De fait, à Zahlé, dans un premier temps à Saida et lors du montage du projet de l'incinérateur à Bikfaya, l'intégration de plusieurs municipalités dans un système de gestion commun des déchets rapproche des municipalités voisines et pourrait amener de la concorde. Toutefois, aucun des trois cas ne donne à voir l'émergence d'un projet de territoire politique commun suscité par la politique des déchets. À Zahlé, le manque d'intérêt pour un projet de territoire régional commun s'explique par les divisions ville centre/banlieues, renforcées par les divisions socio-confessionelles. À Saïda, malgré la présence de l'union et l'intérêt manifesté par les maires de l'agglomération pour cette échelle territoriale, les tensions liées à la recherche d'un lieu pour une nouvelle décharge des déchets inertes minent la volonté de coopération. À Bikfaya, l'avortement du projet d'incinérateur laisse la question ouverte sans réponse, sur un territorial local réduit au fief des Kataeb.

\section{Autonomisation du local par rapport aux institutions centrales}

53 La question de l'autonomisation envers l'État central que la gestion des déchets est entendue et construite différemment selon les discours des acteurs et se traduit diversement dans la réalité politique. Les élites de Bikfaya et Zahlé s'expriment bien plus fortement que les acteurs dominants de Saïda en faveur d'une plus grande décentralisation au Liban. De fait, le réseau d'acteurs de Saïda a des relations privilégiées avec des institutions-clés au sein de l'État central. Ces institutions, surtout le $C D R$, ont fourni une aide essentielle lors du démantèlement de la décharge maritime comme pour les autres grands projets de l'équipe municipale. A l'inverse, les acteurs municipaux à Zahlé ou à Bikfaya sont très critiques du fonctionnement des institutions centrales et revendiquent un transfert des prérogatives et ressources vers les autorités locales. Des trois cas étudiés, Zahlé est toutefois la municipalité qui sur deux décennies s'est le plus affirmée face aux institutions centrales, en pilotant outre les déchets, la gestion des eaux usées et des projets en matière de transport. Bikfaya à l'inverse est la plus dépendante des institutions centrales et des transferts du Fonds autonome des municipalités pour pouvoir maintenir ses services.

Dans cette recherche, l'entrée par les instruments d'action locale pour gouverner la gestion des déchets a permis de montrer, derrière une diversité de choix technologiques et administratifs, une volonté des acteurs municipaux d'utiliser l'instrumentation de l'action publique pour s'affirmer localement et nationalement et construire de nouveaux rapports avec la population en vue de légitimer leurs actions. Les déchets façonnent des territoires politiques dont, néanmoins, la fragilité et l'instabilité doivent être soulignés. Cela renvoie à la nécessité d'autres recherches, notamment axées sur la matérialité des déchets et leur métabolisme, et sur les assemblages socio-techniques en évolution aujourd'hui autour des enjeux de valorisation et de circularité écologique. 


\section{Annexe méthodologique} d'entretiens semi-directifs complètent les 14 entretiens et observations menés dans ces trois villes par les étudiants. Ces entretiens se sont focalisés sur la dimension politique notamment les questions de la décentralisation/autonomisation des municipalités, de la relation aux acteurs politiques nationaux, de la place des habitants et de leur implication, de la relation au secteur privé, des perspectives de développement futur des infrastructures ainsi que les sujets de controverses en lien à ces infrastructures notamment dans le cas de Saida. En plus des représentants municipaux, les entretiens ont inclus des représentants d'organismes de financement concernés, des organisations non-gouvernementales locales ou nationales et des activistes et spécialistes appuyant ou contestant les projets municipaux.

Précisons qu'il a été impossible d'accéder à des informations directes sur les contrats entre acteurs publics et privés: nos informations proviennent ici des entretiens et d'articles de presse. Par ailleurs, les données quantitatives sont au Liban généralement peu fiables en raison de la faiblesse de l'appareil statistique public (Verdeil, Faour, Hamzé (éd.), 2016) et de la diversité des méthodes de production des chiffres présentés par les autorités locales.

BLUHM M., 2007, « New Zahle plant means cheaper waste treatment costs », The Daily Star Newspaper, 23 mars. 


\section{BIBLIOGRAPHIE}

ABBAS I. I., CHAABAN J. K., AL-RABAA A.-R., SHAAR A. A., 2017, Solid Waste Management in Lebanon: Challenges and Recommendations, Journal of Environment and Waste Management, vol. 4, $\mathrm{n}^{\circ}$ 3, p. 235-243.

ABI AKL Y., 2018, Loin de CEDRE, le Parlement avalise la loi nationale sur la gestion des déchets, L'Orient-Le Jour, < https://www.lorientlejour.com/article/1135846/loin-de-cedre-le-parlementavalise-la-loi-nationale-sur-la-gestion-des-dechets.html > (consultation le 25 septembre 2018)

ABIYAGHI M.-N., CATUSSE M., YOUNES M., 2017, From isqat an-nizam at-ta'ifi to the Garbage Crisis Movement: Political Identities and Antisectarian Movements, in DI PERI R., MEIER D. (éd.), Lebanon Facing The Arab Uprisings: Constraints and Adaptation, London, Palgrave Macmillan UK, p. 73-91. < http://dx.doi.org/10.1057/978-1-352-00005-4_5 > (consultation le 18 juillet 2019)

ABOU AFLA R., 2016, Saida's Garbage Experience: How a Mountain Became a Landfill, Legal Agenda. 6 octobre 2016, < http://g6p.002.myftpupload.com/saidas-garbage-experience-how-a-mountainbecame-a-landfill/ > (consultation le 6 janvier 2021)

ABU RISH Z., 2015, Garbage Politics, Middle East Research and Information Project, $\mathrm{n}^{\circ}$ 277, < http:// merip.org/mer/mer277/garbage-politics > (consultation le 21 mars 2016)

AKIKI V., FARFOUR H., 2017, والم-بـالجملة-محارق-اللامركزيّ-الحلّAlhal allamarkazi: mahariq bel jemle wa almfaraq [ La solution « décentralisée » des incinérateurs en série], Al Akhbar, 17 février, > https://hounaloubnan.com/والم-بـالجملة-محارق-اللامركزيّ-الحلّ >

AL BINAA, 2018, بلديات صور تطّلع على تجربة زحلة في التخلص من النفايات Al-binaa Newspaper, > https://www.al-binaa.com/archives/article/205559 > (consultation le 11 janvier 2021)

AL CHARQ, 2015, Wafed baladiyet Trablous fi Saida lilitlaa aala tajribat baladyatiha fi altakhalous min alnifayat [Une délégation de la municipalité de Tripoli à Saida pour en savoir plus sur l'expérience de son municipalité avec les déchets], Al Charq, 3 août.

AL DIYAR, 2016, Fi rad aala bayan jamiiat Min ajl baladi alakhdar ; AlSaoudi : Saida wa maamal alnifayat bikheir [En réponse à la déclaration de l'association Min ajl baladi AlAkhdar ; Saoudi : Saida et l'usine de traitement se portent bien] ", Al Diyar, 3 avril.

AL MOUSTAQBAL, 2016, AlSaoudi aan mahrajanat Saida : hadamna jidar alkhaouf [AlSaoudi sur le Festival de Saida : Nous avons brisé le mur de peur], Al Moustaqbal, 19 septembre.

ANTONIOS Z., 2018, Saïda en rogne contre les déchets, l'eau et l'électricité, L'Orient-Le Jour, < https://www.lorientlejour.com/article/1123703/saida-en-rogne-contre-les-dechets-leau-etlelectricite.html > (consultation le 4 juillet 2018)

ATALLAH S., 2015a, Liberate the Municipal Fund from the Grip of Politicians, < http://lcpslebanon.org/featuredArticle.php?id=52 > (consultation le 1 octobre 2015)

ATALLAH S., 2015b, Garbage Crisis: Setting the Record Straight, LCPS, < http://www.lcpslebanon.org/featuredArticle.php?id=48 > (consultation le 16 août 2015)

ATALLAH S., 2015c, Garbage Crisis Exposes Arrogance and Conflict Among the Political Elite, < http://www.lcps-lebanon.org/featuredArticle.php?id=50 > (consultation le 6 septembre 2015)

AZZI E., 2017, Waste Management Systems in Lebanon : The benefits of a waste crisis for improvement of practices, < http://www.diva-portal.org/smash/record.jsf?pid=diva2:1139992 > (consultation le 26 septembre 2017) 
BAAKLINI, 2012, Déchets à Saïda : les négociations sur le prix de la tonne au point mort, L'OrientLe Jour, 9 janvier, < http://www.lorientlejour.com/category/Liban/article/739316/

Dechets_a_Saida+\%3A__les_negociations_sur_le_prix__de_la_tonne_au_point_mort.html > (consultation le 9 janvier 2012)

BAAKLINI S., 2020, Déchets : qui osera tuer la poule aux œufs d'or ?, L'Orient-Le Jour, < https:// www.lorientlejour.com/article/1217405/dechets-qui-osera-tuer-la-poule-aux-oeufs-dor-.html > (consultation le 28 mai 2020)

BAAKLINI S., 2019, Les incinérateurs approuvés en Conseil des ministres, dont un à Beyrouth, L'Orient-Le Jour, < https://www.lorientlejour.com/article/1184322/les-incinerateurs-approuvesen-conseil-des-ministres-dont-un-a-beyrouth.html > (consultation le 29 août 2019)

BAAKLINI S., 2016, Saïda rêvait d'un jardin à la place de son dépotoir : elle l'a désormais ? L'Orient-Le Jour, < http://www.lorientlejour.com/article/982343/saida-revait-dun-jardin-a-laplace-de-son-depotoir-elle-la-desormais.html > (consultation le 23 avril 2016)

BAAKLINI S., 2011, Saïda et ses environs croulent sous les ordures, L'Orient-Le Jour, 12 avril $2011,<$ http://www.lorientlejour.com/category/Liban/article/699559/

Saida_et_ses_environs_croulent_sous_les_ordures.html >

DE BERCEGOL R., GOWDA S., 2018, A new waste and energy nexus? Rethinking the modernisation of waste services in Delhi, Urban Studies, 26 juin 2018 , p. 2297-2314. < http://dx.doi.org/ $10.1177 / 0042098018770592>$

BONNE E., 2014, Vie publique, patronage et clientèle : Rafic Hariri à Saïda, 1e éd. 1995. (Les Cahiers de l'Iremam), Aix-en-Provence, Institut de recherches et d'études sur le monde arabe et musulman, 108 p. < http://books.openedition.org/iremam/2811 > (consultation le 21 août 2015)

BOUTROS J., 2015, Garbage Crisis in Lebanon - 1997: Same Policy, Repeated History, Legal Agenda. 15 octobre, < http://g6p.002.myftpupload.com/garbage-crisis-in-lebanon-1997-same-policyrepeated-history/ > (consultation le 30 novembre 2020)

CARLES J., 2018, La décentralisation versus technocratisation, in GUIGNARD D., REGOURD S. (éd.), La décentralisation 30 ans après, Toulouse, Presses de l'Université Toulouse 1 Capitole, p. 229-239. < http://books.openedition.org/putc/1397 > (consultation le 20 novembre 2020)

CATUSSE M., KARAM K., LAMLŪM U., 2011, Métamorphose des figures du leadership au Liban : champs et contrechamps des élections législatives de 2009, Beyrouth, Liban, Presses de l'ifpo, 319 p.

CAVÉ J., 2015, La ruée vers l'ordure : conflits dans les mines urbaines de déchets, Rennes, Presses Universitaires de Rennes, xxxii-250 p.

CHAABAN J., 2017, Lebanon's Garbage Crisis: It Still Smells of Corruption - Beirut Today, Beirut Today. 23 août $2017,<$ http://bey-times.com/2017/08/23/lebanons-garbage-crisis-it-still-smells-ofcorruption/ > (consultation le 21 septembre 2017)

CHAMSEDDINE H., 2018, Tajribat bladiyat Saida min alnahia alqanounia [L'expérience de la municipalité de Saida de point de vue juridique], in Alnifayat fi Lubnan : altiqanyat wa kharitat altariq [Les déchets au Liban : les techniques et la feuille de route], Beyrouth, Ordre des ingénieurs et architectes de Beyrouth, p. 70-72.

CHAOUL M., 2012, Zahlé : de la za âma nationale à la za âma dépendante, in MERMIER F., MERVIN S. (éd.), Leaders et partisans au Liban, Karthala/IFPO, p. 435-459. < https://www.cairn.info/leaderset-partisans-au-liban--9782811105952-page-435.htm > (consultation le 20 novembre 2020)

CIRELLI C., FLORIN B., TROIN F. (éd.), 2015, Sociétés urbaines et déchets : éclairages internationaux, Tours, Presses universitaires François Rabelais, $450 \mathrm{p}$. 
CIRELLI C., MACCAGLIA F., MELÉ P., 2017, « L'incinérateur est trop près, la poubelle trop loin » : gérer les déchets en régime de proximité, Flux, 8 décembre, n 109-110, p. 61-72. < http:// dx.doi.org/10.3917/flux1.109.0061 >

DAHCHÉ M., 2015, Algemayel min ma3mal alfarz fi Saida : Li mouaalaja taria lil nifayat [AlGemayel de l'usine de tri de Saida : pour un traitement urgent des déchets], Al Balad, 13 août 2015 ,

DE BERCEGOL R., CAVÉ J., DURAND M., 2018, Informal Recycling vs municipal Waste Service in Asian cities : Opposition or Integration ?, AFD Research Papers, < https://www.afd.fr/en/informalrecycling-vs-municipal-waste-service-asian-cities-opposition-or-integration $>$ (consultation le 10 mai 2019)

DEMARIA F., SCHINDLER S., 2016, Contesting Urban Metabolism: Struggles Over Waste-to-Energy in Delhi, India: Contesting Urban Metabolism, Antipode, mars, vol. 48, n², p. 293-313. < http:// dx.doi.org/10.1111/anti.12191 >

DUBRESSON A., JAGLIN S., 2005, Gouvernance, régulation et territorialisation des espaces urbanisés, in ANTHEAUME B., GIRAUT F. (éd.), Le territoire est mort, vive les territoires ! : Une (re)fabrication au nom du développement, Marseille, IRD Éditions, p. 337-352. < http:// books.openedition.org/irdeditions/3412 > (consultation le 6 janvier 2021)

EL RICHANI D., 2017, This is Not a Revolution: The Sectarian Subject's Alternative in Postwar Lebanon, Thesis, Université d'Ottawa / University of Ottawa, < http://dx.doi.org/http://dx.doi.org/ 10.20381/ruor-20295 > (consultation le 18 juillet 2019)

FAKOYA M. B., 2014, Institutional Challenges to Municipal Waste Management Service Delivery in South Africa, Journal of Human Ecology, 1 février, vol. 45, n² 2, p. 119-125. < http://dx.doi.org/ $10.1080 / 09709274.2014 .11906685>$

FARAH J., GHADDAR R., NASR E., NASR R., WEHBI H., VERDEIL É., 2019, Solid Waste Management in Lebanon: Lessons for Decentralisation, Beirut, Democracy Reporting International, 40 p. < https:// democracy-reporting.org/wp-content/uploads/2019/11/DRI-LEB-

DE_SWM_16102019_english_high.pdf >

FAVIER A. (éd.), 2001, Municipalités et pouvoirs locaux au Liban, (Les Cahiers du CERMOC, ISSN 1607-0488 ; 24). Beyrouth, Centre d'études et de recherches sur le Moyen-Orient contemporain, $438 \mathrm{p}$.

GHADBAN S., SHAMES M., ABOU MAYALEH H., 2017, Trash Crisis and Solid Waste Management in Lebanon-Analyzing Hotels' Commitment and Guests' Preferences, Journal of Tourism Research \& Hospitality, vol. 6, $\mathrm{n}^{\circ}$ 3. < http://dx.doi.org/10.4172/2324-8807.1000171 >

HALPERN C., LASCOUMES P., LE GALÈS P. (éd.), 2014, L'instrumentation de l'action publique : controverses, résistances, effets, Paris, SciencesPo les Presses, 520 p.

HAMMOUD A., KASSEM M., MOURTADA A., 2014, Solid waste to energy strategy in Lebanon: Potential, technology and design, in International Conference on Renewable Energies for Developing Countries 2014, International Conference on Renewable Energies for Developing Countries 2014, p. 75-81.< http://dx.doi.org/10.1109/REDEC.2014.7038535 >

HARB M., 2018, « New Forms of Youth Activism in Contested Cities: The Case of Beirut », The International Spectator, 3 avril, vol. 53, $\mathrm{n}^{\circ}$ 2, p. 74-93. < http://dx.doi.org/ 10.1080/03932729.2018.1457268 > 
HARB M., ATALLAH S., 2015, Lebanon: A Fragmented and Incomplete Decentralization, in HARB M., ATALLAH S. (éd.), Local Governments and Public Goods: Assessing Decentralization in the Arab World, Beirut, LCPS and OSI, p. 189-228.

HUMAN RIGHTS WATCH, 2017, “As If You're Inhaling Your Death" The Health Risks of Burning Waste in Lebanon, New York, Human Rights Watch, 79 p. < https://www.hrw.org/sites/default/files/ report_pdf/lebanon1117_web_1.pdf >

HUYBRECHTS É., VERDEIL É., 2000, Beyrouth entre reconstruction et métropolisation, Villes en parallèle, $\mathrm{n}^{\circ}$ 32-33, p. 83-103.

JAGLIN S., DEBOUT L., SALENSON I. (éd.), 2018, Du rebut à la ressource : Valorisation des déchets dans les villes du Sud, Paris, Agence Française de Développement, 296 p.

JAY M., 2019, Growing Link between Lebanon's Cancer Surge and EU Abetted Corruption, International Policy Digest, 11 mai, < https://intpolicydigest.org/2019/05/11/growing-link-between-lebanon-scancer-surge-and-eu-abetted-corruption/ > (consultation le 20 mai 2019)

KADIRBEYOĞLU Z., SÜMER B., 2012, The Neoliberal Transformation of Local Government in Turkey and the Contracting Out of Municipal Services: Implications for Public Accountability, Mediterranean Politics, vol. 17, n 3, p. 340-357. < http://dx.doi.org/10.1080/13629395.2012.725301

KARAKI J. W., 2016, Transforming Municipal Solid Waste into a sustainable material and energy in Lebanon (Saida), Master of Science in Sustainable Urban Development, College Kellog, Oxford University, $115 \mathrm{p}$.

KATUSIIMEH M. W., MOL A. P. J., BURGER K., 2012, The operations and effectiveness of public and private provision of solid waste collection services in Kampala, Habitat International, vol. $36, \mathrm{n}^{\circ} 2$, p. 247-252. < http://dx.doi.org/10.1016/j.habitatint.2011.10.002 >

KHALIL A., 2015, Fateh mounaqassa li mouraqabat maamal moualajat nifayat Saida [Appel d'offre pour la supervision de l'usine de traitement de Saida], Al Akhbar, 16 octobre.

KHALIL A., 2018, Maamal nifayat Saida : aldawla toulahiq almouhtajin [L'usine des déchets de Saida : l'État poursuit les manifestants], Al Akhbar, 4 juillet $2018,<$ https://al-akhbar.com/ >Community/253477/معمل-نفايات-صيدا-الدولة-تلاحق-المحتجي >

KNOEPFEL P., LARRUE C., VARONE F., 2006, Analyse et pilotage des politiques publiques, (Analyse des politiques publiques, 2), Munich, Bâle, Zürich, Helbing \& Lichtenhahn.

KRAIDY M. M., 2016, Trashing the sectarian system? Lebanon's "You Stink" movement and the making of affective publics, Communication and the Public, 1 mars, vol. 1, $\mathrm{n}^{\circ} 1$, p. 19-26. < http:// dx.doi.org/10.1177/2057047315617943 >

LASCOUMES P., LE GALÈS P. (éd.), 2004, Gouverner par les instruments, Paris, les Presses Science Po, $370 \mathrm{p}$.

LASCOUMES P., SIMARD L., 2011, L'action publique au prisme de ses instruments, Revue française de science politique, vol. 61, $\mathrm{n}^{\circ}$ 1, p. 5. < http://dx.doi.org/10.3917/rfsp.611.0005 >

LE GALÈS P., 2019, « Gouvernance », in Dictionnaire des politiques publiques, Presses de Sciences Po, < https://www-cairn-info.acces-distant.sciencespo.fr/dictionnaire-des-politiquespubliques--9782724625110-page-297.htm > (consultation le 23 février 2020)

MACCAGLIA F., CIRELLI C., 2019, Penser le politique par les déchets, Géocarrefour, 5 juillet, < http://journals.openedition.org/geocarrefour/12663 > (consultation le 5 janvier 2021) 
MAKHZOUMI J., AL-SABBAGH S., 2018, Landscape and Urban Governance: Participatory Planning of the Public Realm in Saida, Lebanon, Land, juin, vol. 7, n 2, p. 48. < http://dx.doi.org/10.3390/ land7020048 >

MANSOUR F., 2018, From Trash Dump to Dreamland: An Entangled History of Toxicity and Capital, Jadaliyya, 13 novembre 2018 , < https://www.jadaliyya.com/Details/38158/From-Trashto-Dreamland-An-Entangled-History-of-Toxicity-and-Capital > (consultation le 2 décembre 2020)

MASSOUD M. A., MOKBEL M., ALAWIEH S., YASSIN N., 2019, « Towards improved governance for sustainable solid waste management in Lebanon: Centralised vs decentralised approaches ", Waste Management and Research, vol. 37, n 7, p. 686-697. < http://dx.doi.org/ 10.1177/0734242X19836705 >

MERMIER F., MERVIN S., 2012, Leaders et partisans au Liban, Paris, France, Liban, Karthala : IISMM, $495 \mathrm{p}$.

MIDDLE EAST STRATEGIC PERSPECTIVES, 2015, Liban : la crise des déchets qui cache une crise du système, in Middle East Strategic Perspectives, < https://www.mesp.me/2015/08/23/liban-la-crisedes-dechets-le-bout-de-liceberg-qui-cache-une-crise-du-systeme/ > (consultation le 20 novembre 2020)

MORSI R. Z., SAFA R., BAROUD S. F., FAWAZ C. N., FARHA J. I., EL-JARDALI F., CHAAYA M., 2017, The protracted waste crisis and physical health of workers in Beirut: a comparative crosssectional study, Environmental Health, 11 avril, vol. 16, n 1, p. 39. < http://dx.doi.org/10.1186/ s12940-017-0240-6 >

MOUSSI M., 2014, Temps du patrimoine, temps des projets urbains : télescopage et fusions à Jbeil, Saïda, Sour (Liban), Revue des mondes musulmans et de la Méditerranée, 15 novembre, $\mathrm{n}^{\circ} 136$, p. 109-134.

OKOT-OKUMU J., NYENJE R., 2011, Municipal solid waste management under decentralisation in Uganda, Habitat International, 1 octobre, vol. 35, n 4, p. 537-543. < http://dx.doi.org/10.1016/ j.habitatint.2011.03.003 >

OLJ, 2018, Les ordures à nouveau ramassées à Saïda, L'Orient-Le Jour, < https://

www.lorientlejour.com/article/1123598/les-ordures-a-nouveau-ramassees-a-saida.html > (consultation le 2 juillet 2018)

OLJ/AGENCES, 2012, Un incendie énorme dans la décharge de Saïda, la ville suffoque, L'Orient-Le Jour, < http://www.lorientlejour.com/category/Liban/article/776819/

Un_incendie_enorme_dans_la_decharge_de_Saida\%2C_la_ville_suffoque.html > (consultation le 7 septembre 2012)

POST A. E., 2018, Cities and Politics in the Developing World, Annual Review of Political Science, vol. 21, n 1, p. 115-133. < http://dx.doi.org/10.1146/annurev-polisci-042716-102405 >

RAH, 2016, Gemayel inaugurates waste management plant in Bikfaya, Middle East Press Releases. 14 mars, < https://www.middleeastpressreleases.com/gemayel-inaugurates-waste-managementplant-in-bikfaya/ > (consultation le 8 janvier 2020)

RAIDY G., 2020, How to Turn Garbage Into Funds for Your Town: The Biclean Example, Medium, < https://ginosblog.com/how-to-turn-garbage-into-funds-for-your-town-the-bicleanexample-205970c1b7b8 > (consultation le 12 mai 2020)

ROZELIER M., 2010, Dossier Déchets, Le Commerce du Levant, novembre, p. 55-67. 
ROZELIER M., 2018, Les nouveaux rois des déchets, Commerce du Levant, < https://

www.lecommercedulevant.com/article/28093-les-nouveaux-rois-des-dechets $>$ (consultation le 5 février 2018)

SALEH M., 2015, Taqrir sidaoui aan alkhota : Alsalbiyat tafouq alijabiyat [Un rapport de Saida sur le « plan » : les inconvénients surpassent les avantanges], Al Safir, 14 septembre.

SALMAN L., BAIRD-ZARS B., 2019, From the Fragments Up: Municipal Margins of Maneuver in Syria and Tunisia, Middle East Law and Governance, 24 novembre, vol. 11, n' 2, p. 244-282. < http:// dx.doi.org/10.1163/18763375-01102006 >

SFEIR R., 2015, Azamat alnifayat tandah drassat wa taftaqid tawafouqan wa qararat; Barnamaj aloumam almoutahida: makab Saida namouzajan! [La crise des déchets ramène des études mais manque le consensus et les décisions; Les Nations Unies : La décharge de Saida comme exemple à suivre !], Al Nahar, 11 août 2015 ,

THE MONTHLY, 2015, Interview with Nabil Zantout, General Manager at IBC, The Monthly, 11 septembre, < https://monthlymagazine.com/article-desc_1805_> (consultation le 1 février 2019)

VERDEIL É., 2017, Des déchets aux remblais : imaginaire aménageur, corruption et dérèglements métaboliques à Beyrouth, Jadaliyya Cities, < http://www.jadaliyya.com/pages/index/26876/desdéchets-aux-remblais_imaginaire-aménageur-corr > (consultation le 4 septembre 2017)

VERDEIL É., 2019, Securitisation of urban electricity supply. A political ecology perspective on the cases of Jordan and Lebanon, in YACOBI H., NSASRA M. (éd.), Routledge Micclea Eastern Cities Handbook, Routledge, p. 246-264.

VERDEIL É., FAOUR G., HAMZÉ M. (éd.), 2016, Atlas du Liban. Les nouveaux défis, Beyrouth, Presses de l'Institut Français du Proche-Orient, 112 p.

WEHBE M., 2012, Saida Trash Mountain: The Stench of Corruption, Al Akhbar English, < https:// web.archive.org/web/20121028054313/http://english.al-akhbar.com/content/saida-trashmountain-stench-corruption > (consultation le 10 octobre 2012)

WEHBE M., 2017, Ala altariq min Bairut ila Saida : Tijarat nifayat mourbiha [Sur la route de Beyrouth à Saida : un commerce de déchets florissant], Al Akhbar, 29 juillet 2017 ,

ZAATARI M., 2012, Workers race against time to finish Sidon breakwater before winter, The Daily Star Newspaper - Lebanon, < /News/Local-News/2012/Sep-26/189212-workers-race-against-timeto-finish-sidon-breakwater-before-winter.ashx > (consultation le 26 septembre 2012)

ZANTOUT N., 2018, Tajribat baladiyat Saida [L'expérience de la municipalité de Saida], in Alnifayat fi Lubnan : altiqanyat wa kharitat altariq [Les déchets au Liban : les techniques et la feuille de route], Ordre des ingénieurs et architectes de Beyrouth, p. 44-48.

ZBEED M., 2012, \$ 5 Million a Year: The Tip of the Sukleen Waste Pile, Al Akhbar English, 28 août, < http://english.al-akhbar.com/content/5-million-year-tip-sukleen-waste-pile > (consultation le 28 août 2012)

ZEATER T., 2015, Jawla lilsinaiyin fi maamal farz alnifayat fi Saida [Visite des industriels de l'usine de tri des déchets à Saida], Al Liwaa, 13 août.

\section{NOTES}

1. En moyenne, le FAM représente $31 \%$ des revenus municipaux au Liban (The Lebanese Center for Policy Studies (LCPS), 2015). 
2. Données du CDR ( https://portal.cdr.gov.lb/reports/Foreign-Funding/ LoansAndGrantsRatifiedSignedAndApprovedDistributionBySectorAndFundingSource le 30/11/2020)

3. Dans un entretien avec des représentants de USAID (18 janvier 2017) et des échanges informels avec des agences de l'ONU (UNHABITAT) et des ONGs internationales (ACTED), M. Zoughaib a été loué pour son « sérieux et professionalisme».

4. Forme d'intercommunalité qui regroupe plusieurs municipalités d'un même district.

5. Le maire déclarait au journal The Daily Star : "Nobody was aware in the past of the harm that is being done to the environment. The cost of these misuses of the environment was much higher than the cost of treating the waste. We paid the price with our health. We don't know what harm we have done to ourselves in the past 30,40 years. » (Bluhm, 2007).

6. Dans un texte récent sur le site municipal, la municipalité exprime ses réticences par la difficulté d'assurer le niveau de suivi demandé pour contrer les risques environmentaux des incinérateurs, notamment dans l'éventualité d'un changement de majorité au sein du conseil. Le texte mentionne un procès pour la production de l'essence pour voiture à partir des déchets qui serait une alternative crédible

7. Le site reçoit des visites répétées de délégations de tout au Liban (e.g. AlBinaa, 2018)

8. Plus précisément la municipalité cède la propriété à $I B C$ à condition d'usage unique, le traitement des déchets de l'union Saida-AlZahrani

9. Ce discours envers l'usine est même repris par les experts du PNUD présentant le projet comme un modèle à suivre (Sfeir, 2015).

10. Entretien avec le membre du conseil de l'Union en charge de la préparation du plan stratégique le 02/03/2018.

11. (voir les propos de militants proches du député nassérien Saad in Karaki 2016, p. 62-63)

12. Ce marché est attaqué dans la presse pour corruption (Wehbe, 2017).

13. Très médiatisés sur la page Facebook de Biclean.

14. «ils (Sakiet El Misk- Bhersaf) viennent de se retirer car l'Etat ne leur verse plus (comme à toutes les autres municipalités) leur part du fond indépendant des municipalités (FIM). Ils préfèrent donc passer par les services de RAMCO payé par le CDR, même si cela correspond en fait à un cout très élevé, qui se traduira à terme par une baisse des fonds qui leur seront alloués par le FIM. Dans l'immédiat, ils n'ont pas à payer Bikfaya pour le service. Sans compter que RAMCO jette tout à la mer, alors que nous on trie, on recycle, on envoie les non recyclables dans des usines... ». Entretien avec Nicole Gemayel, le 17/1/2019.

15. Projet CEDRE $n^{\circ} 37355 \mathrm{NF}$ La crise des déchets au Liban: controverses et nouvelles formes de gestion urbaine (2017-2019)

\section{RÉSUMÉS}

Cet article étudie la capacité des autorités locales à stabiliser une gouvernance des déchets et les effets politiques de cette transformation de l'action publique locale. Le cadre analytique s'inscrit dans la sociologie des instruments de politique publique et suit les politiques que les pouvoirs municipaux mettent en place sur le terrain pour faire face à un problème urgent. L'étude porte sur le Liban en raison de la crise des déchets en cours, et toujours en suspens, qui a donné lieu à 
de vastes protestations. Plus précisément elle porte sur trois municipalités en dehors ou aux marges de la région de la capitale, Saïda, Zahlé et Bikfaya, où différents types de solutions innovantes (technologiquement et/ou politiquement) ont été mises en œuvre. Là, nous documentons l'élaboration des politiques de gestion municipale des déchets solides en étudiant les contrats, le financement, la communication, l'aménagement urbain, les périmètres d'opération et l'environnement. Nous étudions les effets politiques de la stabilisation de ces assemblages d'instruments de gouvernance du point de vue de la légitimation du pouvoir local, de la construction d'un territoire de coopération local ou régional et les relations entre le pouvoir local et le pouvoir central. La recherche s'appuie sur une analyse de la presse et sur des enquêtes par entretiens dans les localités et les instances de définition de ces politiques publiques. Les résultats montrent que les choix politiques dépendent très fortement du type de réseaux politiques locaux, et du degré d'urgence des temporalités de décision.

This article examines the capacity of local authorities to stabilise the governance of municipal waste and the political effects of this transformation of local public policies. The article adopts an analytical framework inspired by the sociology of public policy instruments and follows the policies municipal powers build on the ground to cope with a pressing issue. We are interested in the case of Lebanon because of the ongoing, and still pending trash crisis that resulted in wide protests. We studied three municipalities outside of the capital region, Saida, Zahle and Bikfaya, where different kind of innovative solutions (technologically and/or politically) have been implemented. There, we document the elaboration of the SWM policies regarding contracting, financing, communication, spatial planning, service districting and environment. We also analyse the political effects of the stabilisation of these assemblages of instruments from the perspective of legitimising of local power actors, the building of a territory of local or regional cooperation and the relationships between the local and the central governments. The research relies on press review and local surveys (interviews). Results show that political choices depend upon the kind of local political networks and the degree of emergency that frames the temporality of decision. In doing so, we carefully read the local and local/national political dynamics that allow, constrain or prevent the policy choices. The findings show that policy choices are very strongly dependent upon the kind of local policy networks. Conversely, they also highlight that the institutional frameworks at the local and national scale leaves them wide margins of manover.

\section{INDEX}

Keywords : municipal waste, municipality, instruments public policy, decentralisation, public service, urban services, infrastructure, participation, Saida, Zahle, Bikfaya

Mots-clés : déchets ménagers, municipalités, décentralisation, urbanisme, instruments politique publique, services publics, services urbains, infrastructures, participation, Liban, Bikfaya, Saïda, Zahlé

\section{AUTEURS}

\section{JIHAD FARAH}

Université Libanaise, Laboratoire CERDA, Beyrouth, Liban jihadfarah@gmail.com

\section{ÉRIC VERDEIL}

Sciences Po, Centre de recherches internationales (CERI), CNRS, Paris, France

eric.verdeil@sciencespo.fr 